\title{
A concept for in-space, system-level validation of spacecraft precision formation flying
}

\author{
Jesse Leitner* J. Russell Carpenter ${ }^{\dagger} \quad$ Bo J. Naasz ${ }^{\ddagger}$ \\ NASA Goddard Space Flight Center, Greenbelt, MD, 20771, USA \\ Asif Ahmed ${ }^{\S} \quad$ Fred Y. Hadaegh ${ }^{\Uparrow} \quad$ Daniel P. Scharf $\|^{\|}$ \\ Jet Propulsion Laboratory, Pasadena, CA, 91109, USA
}

\begin{abstract}
A number of international space agencies and organizations, to include the National Aeronautics and Space Administration (NASA), the European Space Agency (ESA), and the Centre National d'Etudes Spatiales (CNES), to name a few, have embraced the concept of spacecraft formation flying to revolutionize the capabilities of astronomy and Earth remote sensing from space. The concept has been around well over a decade and a wide array of technologies and capabilities have been developed to enable multiple spacecraft to collaborate in a highly-coupled manner as would be required for a formation flying mission. Furthermore, many relevant capabilities for formation flying have been demonstrated in the area of rendezvous and docking, loosely-controlled formations, and in missions with collaborating spacecraft with very precise metrology. However, in considering the case of precision formation flying (PFF), i.e, when the relative geometry of multiple vehicles must be controlled on-board in a continuous and precise manner, there have been several missions proposed, but the realization in space has not yet occurred due to a range of issues. This paper will briefly examine those issues and present a concept for demonstrating a core capability for performing PFF, necessary for virtually any PFF mission concept, that will help to overcome the problems encountered in prior attempts and help to allay the risks to enable future PFF science missions. This paper summarizes a flight demonstration concept development that resulted from NASA's New Millennium Program ST9 Concept Definition Phase.
\end{abstract}

\section{Introduction}

A number of international space agencies and organizations, to include the National Aeronautics and Space Administration (NASA), the European Space Agency (ESA), and the Centre National d'Etudes Spatiales (CNES), to name a few, have embraced the concept of spacecraft formation flying to revolutionize the capabilities of astronomy and Earth remote sensing from space. The concept has been around well over a decade and a wide array of technologies and capabilities have been developed to enable multiple spacecraft to collaborate in a highly-coupled manner as would be required for a formation flying mission. Furthermore, many relevant capabilities for formation flying have been demonstrated in the area of rendezvous and docking, ${ }^{1}$ loosely-controlled formations, ${ }^{2}$ and in missions with collaborating spacecraft with very precise metrology. ${ }^{3}$ However, in considering the case of precision formation flying (PFF), i.e, when the relative geometry of multiple vehicles must be controlled on-board in a continuous and precise manner, there have been several missions proposed, but the realization in space has not yet occurred due to a range of issues. This paper will briefly examine those issues and present a concept for demonstrating a core capability for

*Lead Engineer for Distributed Space Systems, Code 591, Associate Fellow AIAA.

${ }^{\dagger}$ Navigation Lead, Code 595, Associate Fellow AIAA.

$\ddagger$ Aerospace Engineer, Code 595.

§Senior Engineer, Guidance and Control Analysis Group, 4800 Oak Grove Rd

"Supervisor, Guidance and Control Analysis Group, 4800 Oak Grove Rd, Fellow AIAA.

"Senior Engineer, Guidance and Control Analysis Group, 4800 Oak Grove Rd, Member AIAA. 
- performing PFF, necessary for virtually any PFF mission concept, that will help to overcome the problems encountered in prior attempts and help to allay the risks to enable future PFF science missions.

\section{I.A. Background}

A recent concept study at NASA has resulted in the following conclusions which have given rise to the approach described in this paper: (1) there are many sensor technologies available or in advanced development that meet component-level requirements for future PFF missions (2) the cost of flight units on such technologies and the impacts on spacecraft requirements bring them out of reach for a highly cost- constrained technology demonstration mission and (3) the major gap in PFF is not so much in the component technologies, but in the system that integrates all of the component technologies together (sensors, actuators, algorithms, software, and communications) and performs relevant PFF operations. Hence we place more emphasis on PFF as a system-level capability than on the specific performance numbers that are mostly dependent on high-cost components. This approach will help avoid many of the cost and performance issues that have led to the demise of previous PFF mission concepts.

\section{I.A.1. Paper Organization}

This paper will present some key elements of a low-cost, low-risk option to demonstrate the critical capabilities required for any future space mission requiring PFF. First, we will provide a mission overview and description including some of experiment and operations. Next we will present results on navigation and relative navigation analysis to indicate what type of performance may be achievable under tight cost and orbital placement constraints. Furthermore, we will describe the formation and attitude control system architecture, applicable to the full range of future PFF missions, as applied to this demonstration concept. Finally, we will describe mission studies and technology validation on the ground in the Formation Flying Test Bed (FFTB) at Goddard Space Flight Center (GSFC).

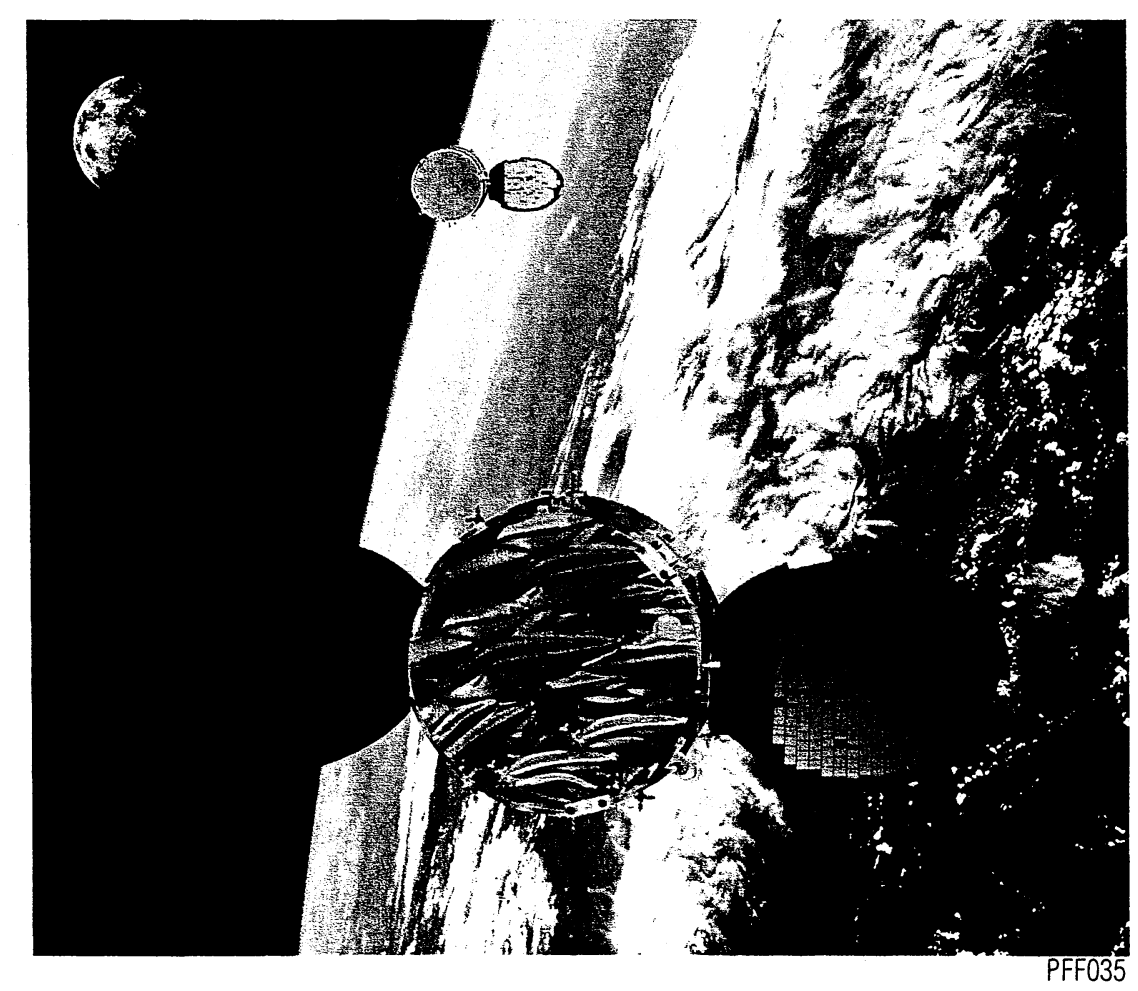

Figure 1. Two Spacecraft Formation Flying Concept 


\section{- I.A.2. Context}

This paper provides a brief overview of some of the results of a Phase A study performed under the auspices of NASA's New Millennium Program, as part of the Space Technology - 9 (ST9) Concept Definition Phase. Within ST9, five technology concept areas competed for a low-cost technology validation flight, the other areas being solar sail, large space telescope, precision landing, and aerocapture technologies. In the end, the resulting budget for the project was reduced significantly, which subsequently eliminated PFF and several of the other concepts from the prospect to continue on for Phase B.

\section{I.A.3. Establishing relevance and value of a PFF demonstration mission}

In consideration of the problem of precision formation flying, and specifically how to validate key elements and buy down risk for future missions on a very tight budget, we can look at layers of formation flying capability that would be undertaken as funding permits, in order of criticality. Each additional layer adds a new "order," so to speak, of capability at a substantial increment of cost. One view is as follows:

1. Two or more spacecraft-two is minimum to demonstrate any form of formation flying, while four spacecraft or more enable a three-dimensional formation

2. Integration of intersatellite communication, on-board relative measurement, and on-board control of relative state-the process of these elements all performing together in a continuous fashion is the crux of $\mathrm{PFF}$

3. Relevant operations-some of the biggest concerns in a multi-spacecraft collaborative mission will naturally lie in the ability to collectively operate so as to enable a distributed instrument or system of instruments

4. Key sensors-clearly the use of sensors in a demonstration mission that would later be used operationally goes a long way to allay real and perceived risks; however, this layer can drive the costs and risks up on a demonstrator, both directly and indirectly, and in the past, this problem has prevented many PFF demo concepts from making it to space

5. Key actuators-finally, in order to confirm that all relevant and scalable elements of a PFF system have been demonstrated in an integrated fashion, a prototype of the actual thruster and mechanical actuator system would be the final addition to the mission

Figure 2 shows this pictorially with a core, general PFF architecture at the center and with a complex intersatellite communication network for a large number of spacecraft on the outside. While each of the layers above have degrees within, the layers themselves are sufficient to define significant increments of capability that are added at significant elements of cost. The mission architect must first consider the budget available to determine how many layers down are feasible. The next step is to identify the degrees within each layer, based on a cost-benefit analysis. In the figure, the layer that includes sensors with full relative observability represents the outermost layer of a mission concept that would meet minimum capability requirements for a worthwhile PFF demonstration. The layer that includes high precision sensors represents a knee in the curve where the cost and technological risk grow at a much higher rate for a given capability increment. The key factors for this include the cost of precise flight-qualified instruments based on new technologies, the impacts and constraints they impart on the spacecraft design, and the risks that issues might arise in sensor performance while packaging into a format for flight.

\section{Mission Overview}

Considering a highly-cost-constrained, yet risk-averse, environment, we present a mission concept that employs the first six layers shown in Figure 2. We emphasize a general PFF architecture that is both relevant and scalable to a full range of future PFF missions, requiring (1) incorporation of mission-specific sensors and actuators, (2) adherence to interface requirements, (3) incorporation of spacecraft specific parameters and gains associated with required performance characteristics, and (4) for large formations, the incorporation of a large scale communication architecture and hardware and software component set. We will denote this scalable architecture the Formation Flying System (FFS). The FFS comprises three major elements: 


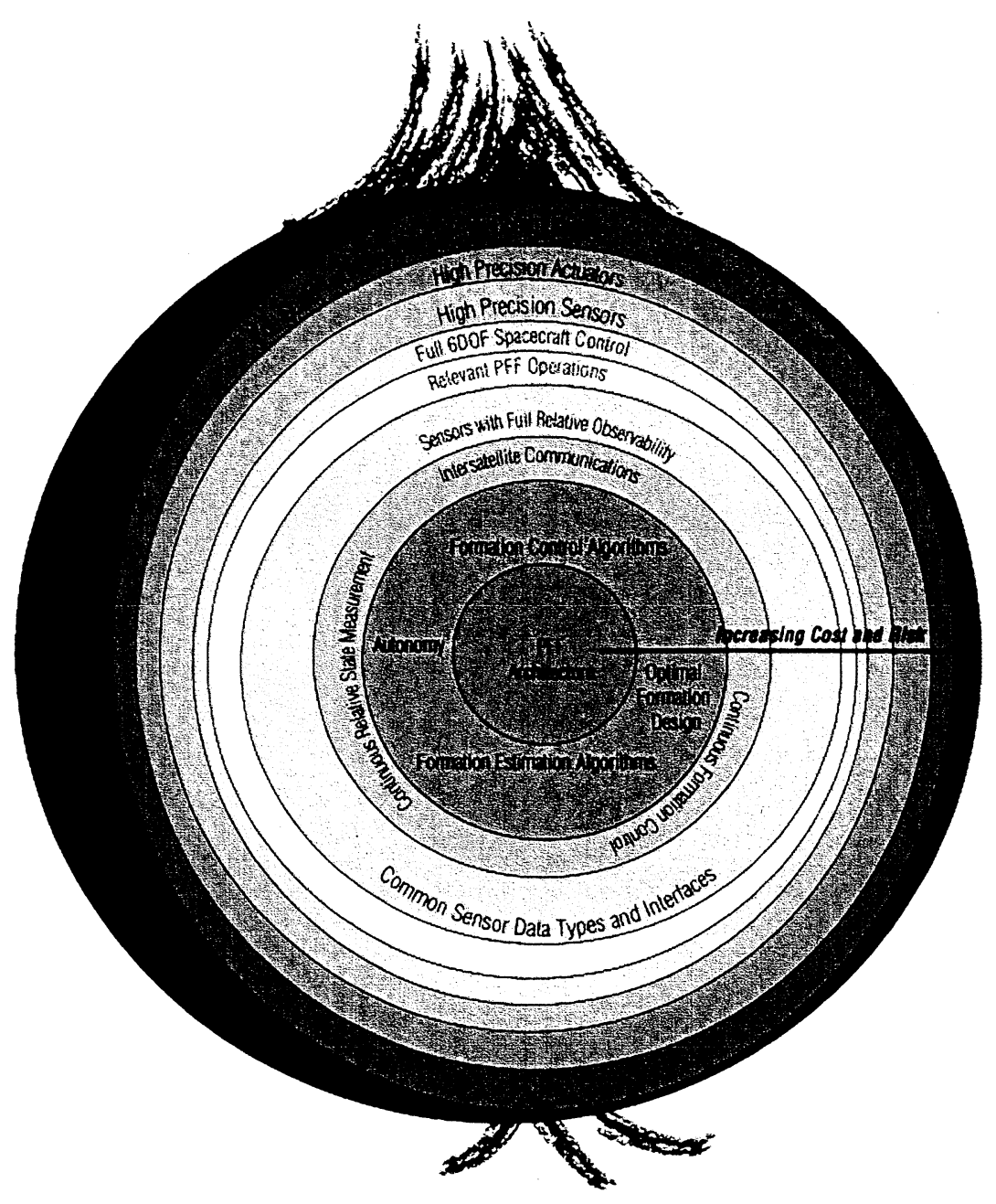

Figure 2. The PFF Onion

a six degree of freedom (6DOF) formation controller, denoted the Formation and Attitude Control System (FACS), a full relative state estimator, the GPS-Enhanced On-board Navigation System (GEONS), and an intersatellite communications and ranging device (ICS). The architecture is shown in Figure 3.

A proposed mission concept will be centered upon performing precision formation flying using spacecraft that include the FFS. Henceforth, we will briefly describe a mission design approach and key experiments in the mission that suitably prove out PFF as a capability that can be performed in space.

All PFF experiments are designed to emulate maneuvers necessary for precision formation flying, science data collection, and formation safety for customers such as the Terrestrial Planet Finder-Interferometer (TPF-I), Stellar Imager (SI), and the Black Hole Imager (BHI). Since ST9 PFF is in LEO, near-Earth orbital effects are treated as dynamic disturbances to be compensated for by the formation control system. Natural, near-Earth, relative orbital dynamics are exploited to varying degrees to efficiently demonstrate a range of operations relevant to future PFF missions. Some formations have been proposed for Earth science, such as distributed interferometric synthetic aperture radar or distributed sparse apertures. Relative orbital dynamics are a significant differential force among a formation of spacecraft, and must be accounted for. ST9 PFF will also demonstrate Jperations relevant to such missions. The PFF experiments include the following formation-flying maneuvers and capabilities:

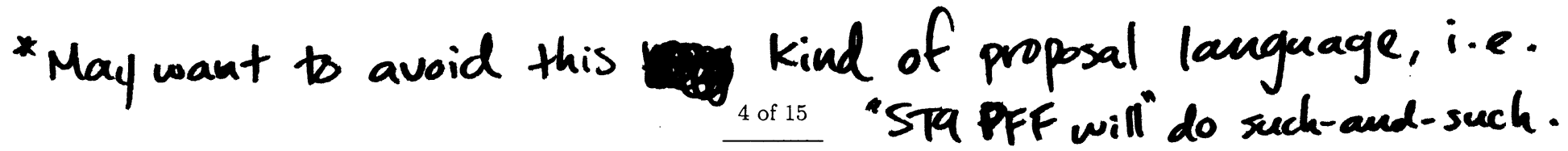




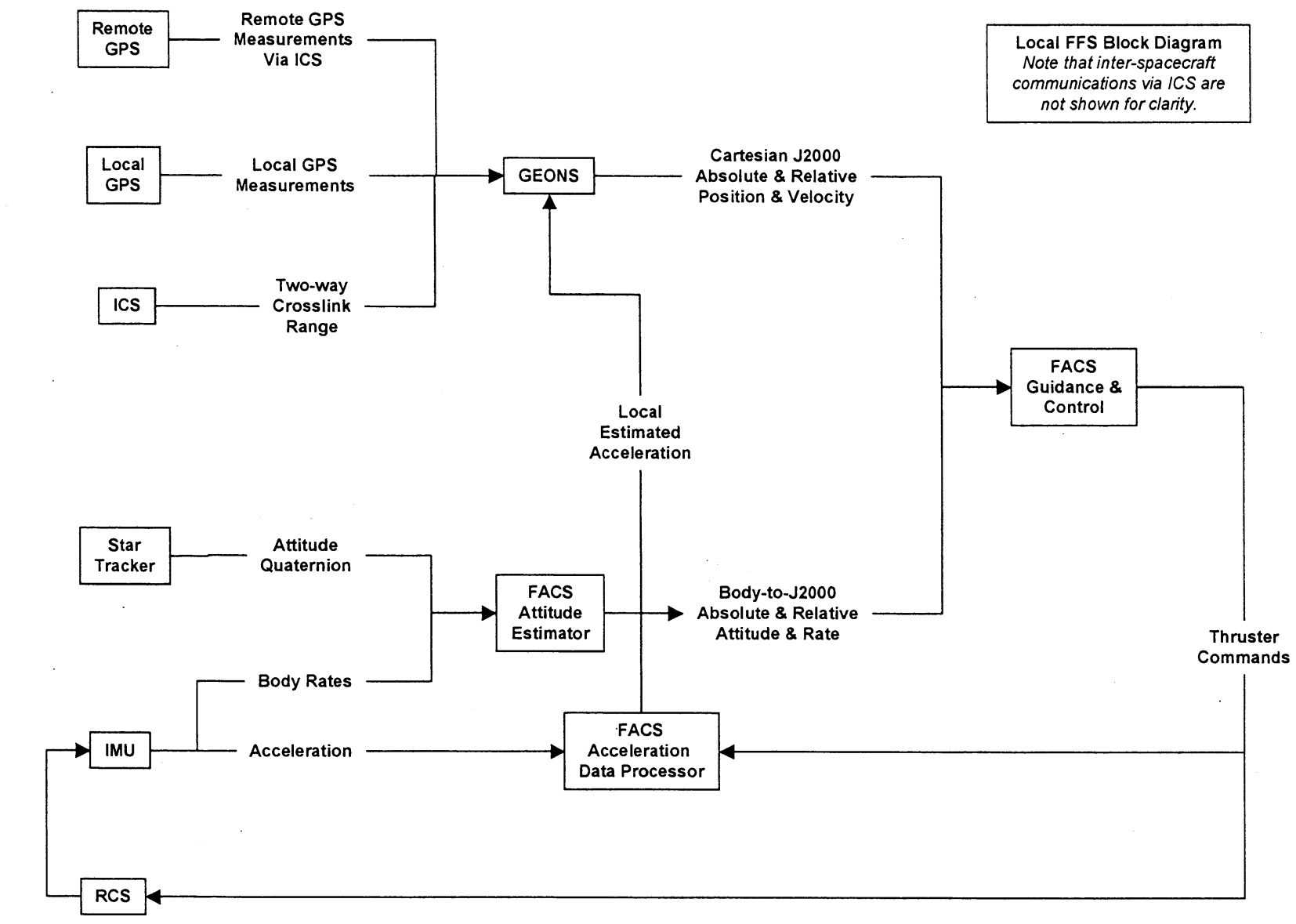

Figure 3. FFS Block Diagram

- Formation Initialization/Acquisition

- Formation Hold

- Formation Reconfiguration, including resizing and passive collision avoidance

- Formation Synchronized Rotations

- Reactive Collision Avoidance

- Graceful Formation Degradation

- Formation Safing

- Formation Dispersal

- Autonomous Observations

The propellant required to execute some experiments in the near-Earth environment necessitates short experiment durations. These capabilities will be demonstrated by repeating experiments for durations of a half orbit. In order to effectively demonstrate PFF over longer durations, other experiments will accommodate the central body gravity of the Earth, but will cancel all other disturbances. Some design guidelines established for the PFF experiments follow. The PFF experiments are not restricted to a particular portion of the orbit. The experiments simulate tasks required for actual PFF missions (most of which are in 
- far-Earth, low-gravitation regimes) by targeting relative motion similar to that required by the PFF missions. Experiments designated as Gravitationally Unconstrained (GU) target relative motion that is nearly identical to the relative motion of a deep-space PFF mission, while experiments designated as Gravitationally Constrained (GC) perform identical tasks with relative motions slightly modified to accommodate the central body gravity. The GC experiments allow extensive testing of PFF architecture, hardware, and algorithms while minimizing fuel consumption. Passive collision avoidance is a goal during the mission. The purpose is to ensure, through careful experiment design, that if the formation were to enter a safe mode at any time during the experiment, the ensuing uncontrolled relative motion will be collision-free. Certain orbit geometries and the need to sun-point during the experiment can prevent experiments from meeting this provision. Changes to the formation orbit should be minimized during a PFF experiment. This ensures a nearly-Keplerian formation orbit and reduces orbit corrections and associated $\Delta V$ penalties. This requirement will be met by: a) requiring all formation spacecraft to have a thruster configuration which imparts (ideally) no net translational impulse to the spacecraft during attitude control; and b) requiring exactly one spacecraft in the formation to not exercise any translation control during an experiment. This non-maneuvering spacecraft is free to exercise attitude control, however. Further, in order to balance fuel consumption across the formation elements, the designation of the nonmaneuvering spacecraft will change from one PFF experiment to the next.

Table 1 shows a list of experiments performed and a mapping of which formation flying primitive elements apply to each.

\section{Relative Navigation}

Navigation will be performed onboard each ST9 PFF vehicle by the GPS-Enhanced Onboard Navigation System (GEONS). ${ }^{4}$ GEONS is flight software developed at NASA GSFC to provide high-accuracy orbit, and time information onboard the spacecraft. The navigation algorithms consist of the folowing core components: 1) an extended Kalman filter (EKF) with physically representative models; 2) a high fidelity state dynamics model; and 3) initialization and enhanced fault detection isolation and recovery (FDIR) capabilities.

The GEONS EKF includes models for a variety of measurements, including: GPS pseudorange, Doppler, and carrier phase; inter-spacecraft crosslink range, Doppler, and line-of-sight vector; TDRSS forward-link Doppler; ground-station-to-satellite range and Doppler; celestial objects measurements (line-of-sight vectors, sun elevation angle, near-to-far- and near-to-near-body pseudoangles); and point solution position measurements.

High-fidelity state dynamics models in GEONS include non-spherical Earth (JGM-2 up to order 30 and degree 30 ) and Lunar (LP100K) gravity models, point mass gravitation models for the sun and planets, atmospheric drag and solar radiation pressure models, and external accelerations expressed in a variety of convenient coordinate systems. In addition to absolute and relative position and velocity states, drag and solar radiation pressure, time bias and drift, unmodeled acceleration, ionospheric path delay, and numerous measurement biases can be estimated in the filter.

In addition to EKF state vector and covariance estimation capabilities, a number of other features are available in GEONS. These features include: "cold-start" filter initialization; automatic FDIR capabilities (including measurement dittino filter convergence monitoring, comparison of filter solution with other available solutions position and velocity covariance tests, etc); real-time state vector propagation, and maneuver argetting a Lambert algorithm.

The various measurement and filter options described above are either included or not included in the desired flight GEONS configuration through build-time options, making the system highly reconfigurable and scalable to a variety af missions. Onboard processor capabilities, and the availability of highly accurate sensors are the onl limittiv factors in the performance of the system. As the number of vehicles estimated, as well as the measurement inputs are flexible in GEONS, this system is fully scalable to future PFF formations.

The ST9 PFF GEONS configuration includes processing of GPS pseudoranges (both standard and singlydifferenced), and two-way crosslink ranges. At present, the PFF spacecraft design includes a General Dynamics (GD) Viceroy GPS receiver on each vehicle, and an Inter-spacecraft Communications System (GD Multi Mode Transceiver plus ranging). Measurements from these devices make up the basis for the GEONS navigation solution performed on each vehicle for both the local vehicle state (absolute position and velocity, clock bias, clock drift, drag coefficient), and the remote vehicle state (relative position and velocity, clock

\section{editing, targeting, limiting}

American Institute of Aeronautics and Astronautics 
bias, clock drift, drag coefficient). Analysis of this navigation architecture in the projected ST9 PFF orbit, and with typical temperature-controlled crystal oscillator (TCXO) receiver clock errors, GPS SV transmitter clock and broadcast ephemeris errors $(2 \mathrm{~m}, 1 \sigma)$, errors from ionospheric delapexpected GPS receiver $(2 \mathrm{~m}$, $1 \sigma)$ and crosslink range $(50 \mathrm{~cm}, 1 \sigma)$ measurement errors have been performe Results from that work show typical relative errors of $0.179 \mathrm{~m}$ RMS in position (time history shown in Figure 4), and $1.9 \mathrm{~cm} / \mathrm{s}$ RMS in velocity (time history shown in Figure 5).

Hardware-in-the-loop (HWIL) testing of the PFF onboard navigation system has also been performed in the Formation Flying Test Bed at GSFC. These tests included Spirent GPS Signal Simulators and Orion GPS receivers developed by the German Space Agency (DLR), and $50 \mathrm{~cm}$ software-simulated crosslink range data. Results from one 18-hour HWIL simulation show typical GEONS relative navigation errors of $0.80 \mathrm{~m}$ $\mathrm{RMS}$ in position, and $0.19 \mathrm{~cm} / \mathrm{s}$.
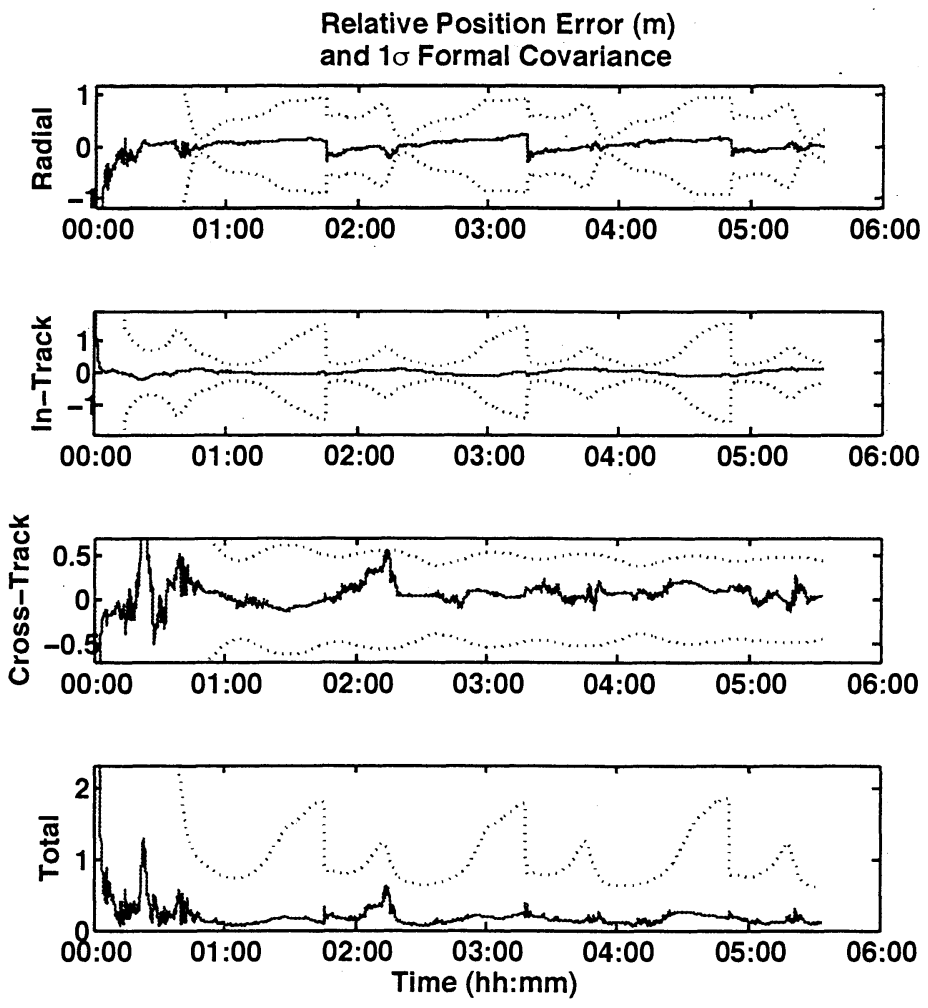

Figure 4. Relative Position Estimates

\section{Formation and Attitude Control System}

The purpose of the FACS is to provide three-axis inertial attitude and interspacecraft range and bearing control among the spacecraft in the formation. Additionally, FACS will provide the capability of initializing and reacquiring the formation through the acquisition of interspacecraft range and bearing knowledge, using the available on-board formation sensing capability. The FACS will ensure collision free operation of the formation throughout all mission phases.'

\section{IV:A. FACS Architecture}

The FACS architecture must be scalable yet robust while encompassing the traditional single spacecraft control functions and capabilities. The overall FACS architecture, regardless of the specific implementation, retains the traditional single spacecraft attitude estimation, attitude guidance, and attitude control functions within each spacecraft in the Standalone mode, while providing centralized formation guidance function on any of the selected spacecraft in formation which is designated the formation leader. These centralized formation guidance functions ensure resource efficient, collision free and coordinated translation commanding 

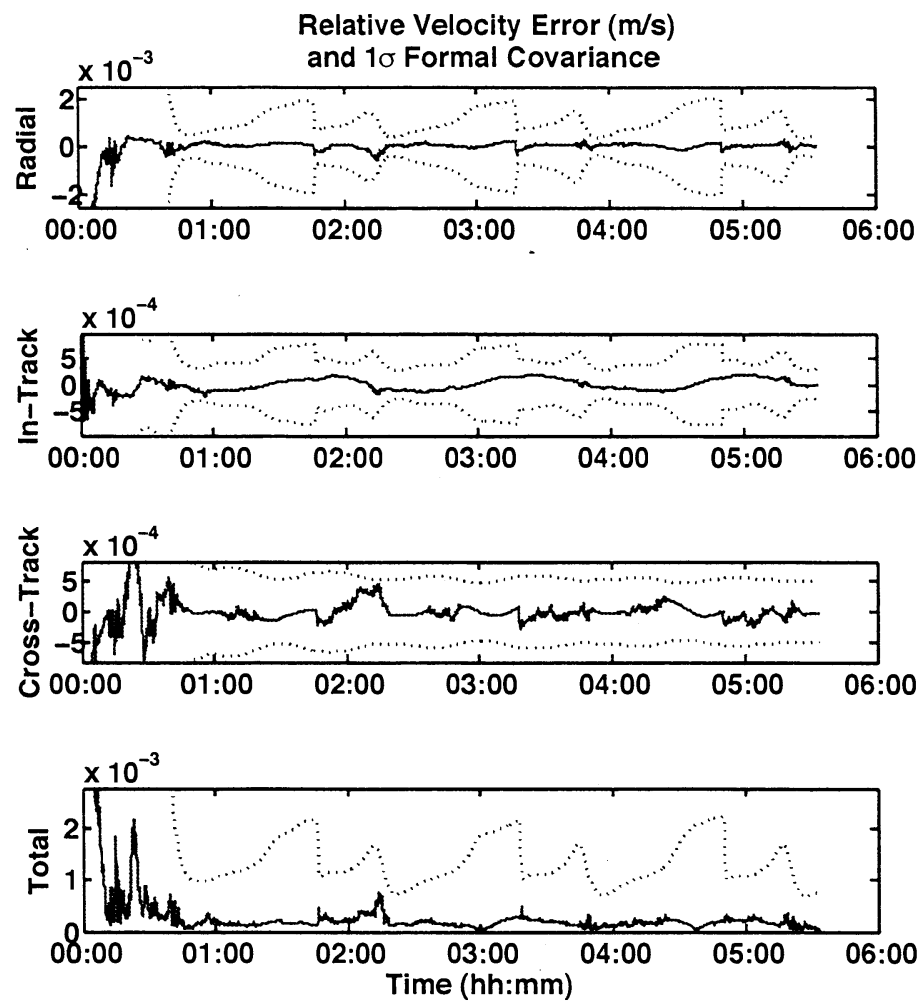

Figure 5. Relative Velocity Estimates

across all spacecraft within the formation via the ICS. During formation experiments, the centralized formation guidance commands, once received over the ICS, are implemented locally on each spacecraft through a local 6DOF control function. In the event of ICS dropouts or failure, each spacecraft within the formation reverts back to local Standalone on-board attitude control mode to maintain power-positive sun-pointing, while still capable of performing ground-commanded linear $\Delta V$ maneuvers.

The overview of the FACS architecture is shown in Figure 6.

Key features of the FACS Architecture are summarized below:

1. Hybrid Control Architecture

Centralized translation guidance

Decentralized attitude guidance

Decentralized translation control

Decentralized attitude control

2. Identical FACS Flight Software load on all spacecraft in the formation

Each spacecraft's FACS software is configured to take on a formation leader/follower role.

\section{IV.B. FACS Algorithms}

\section{IV.B.1. Formation Path Planner}

The path planner performs 6DOF trajectory definition of the spacecraft in formation subject to inertial attitude, minimum spacecraft-to-spacecraft separation for collision avoidance, and actuator capability constraints while minimizing time or fuel usage. ${ }^{5,6}$

\section{IV.B.2. Formation Controller}

The formation control algorithms ensure that the spacecraft and formation follow the inertial attitude and formation range and bearing profiles as prescribed by formation guidance by computing and commanding 


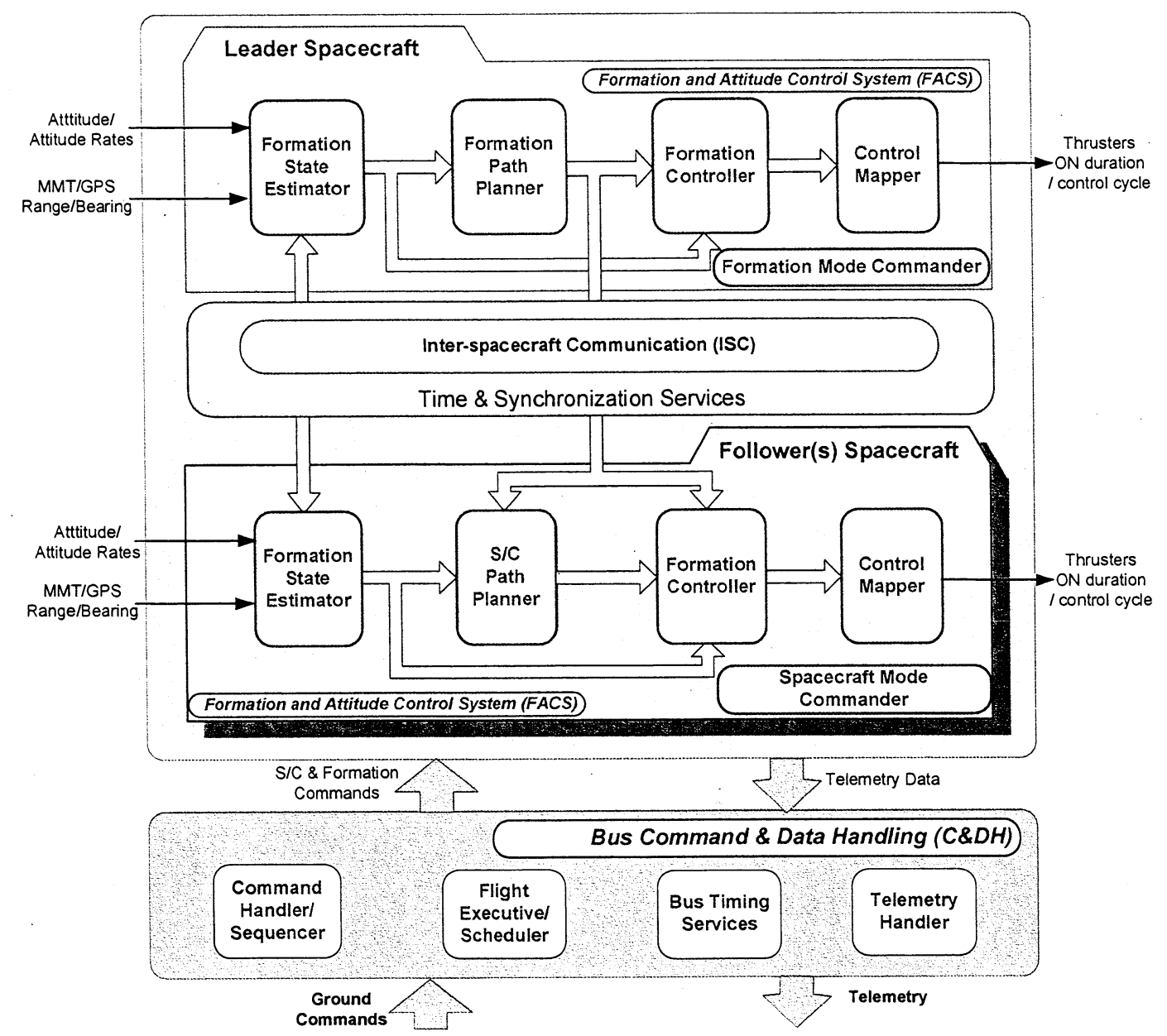

Figure 6. Formation and Attitude Control System Architecture

the needed forces and torques to the bus for mapping to available actuators. The details of the algorithm are provided in.$^{5,7}$

\section{IV:B.3. Mode Commander}

This element is the logical state machine to coordinate all activities and modes of operations of the FACS within each spacecraft and across multiple spacecraft in formation. The Mode Commander has limited fault response capability but no fault identification and isolation capability.

\section{IV.B.4. Control Mapper}

The last element decomposes and allocates the commanded forces and torques from the controller to the available control actuators given their mounting configuration and operational states.

\section{Ground-based Technology Validation}

A key element of the technology validation process of $\mathrm{PFF}$ is ground demonstration, carrying the system technology through TRL levels 5 and 6 . Two critical component technologies required in the integrated process for the ST9 PFF mission are the ICS and the FACS software. The purpose of this validation is to verify the system-level performance with technology components in the loop in a relevant environment, ensuring that the mission requirements can be achieved in a simulation environment with the highest affordable fidelity. Figure 7 shows the block diagram of the ST9 configuration. 


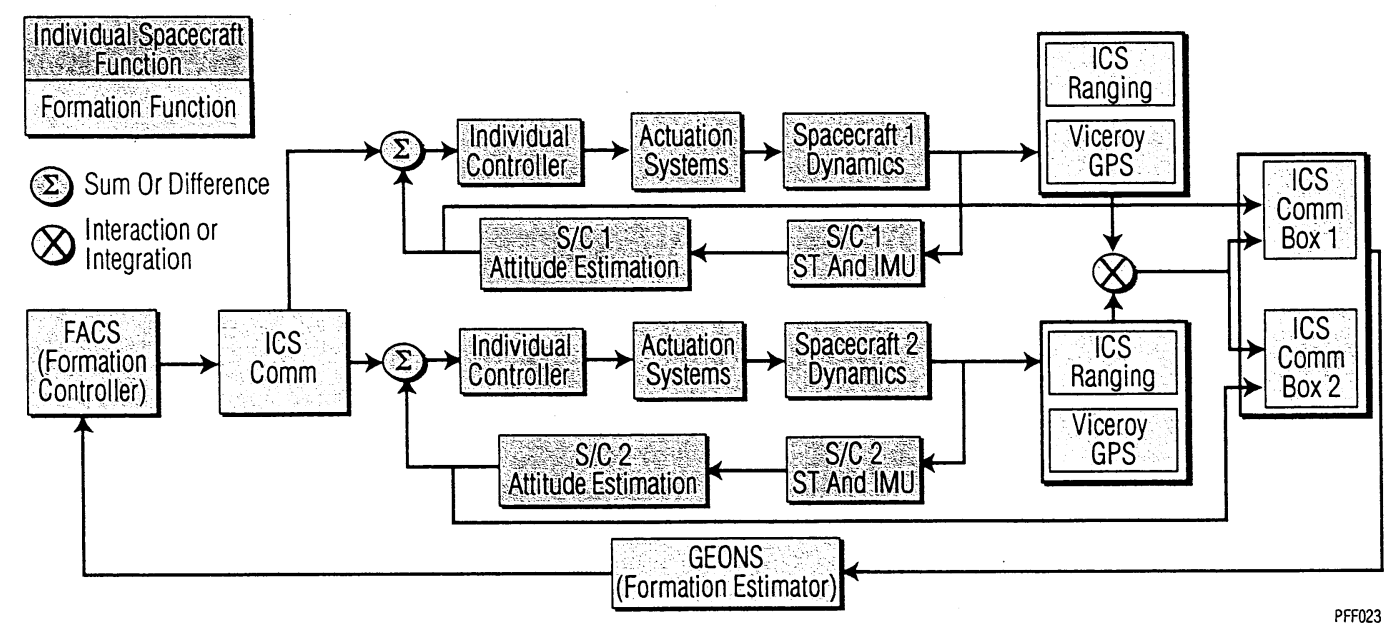

Figure 7. ST9 PFF Block Diagram

The goal of the TRL 5-6 ground testing will be to exercise this configuration using hardware in the loop on the ground. Figure 8 focuses on the specific replication of the block diagram in Figure 7 on the ground, stressing the most important elements of the integrated system to the greatest degree affordable.

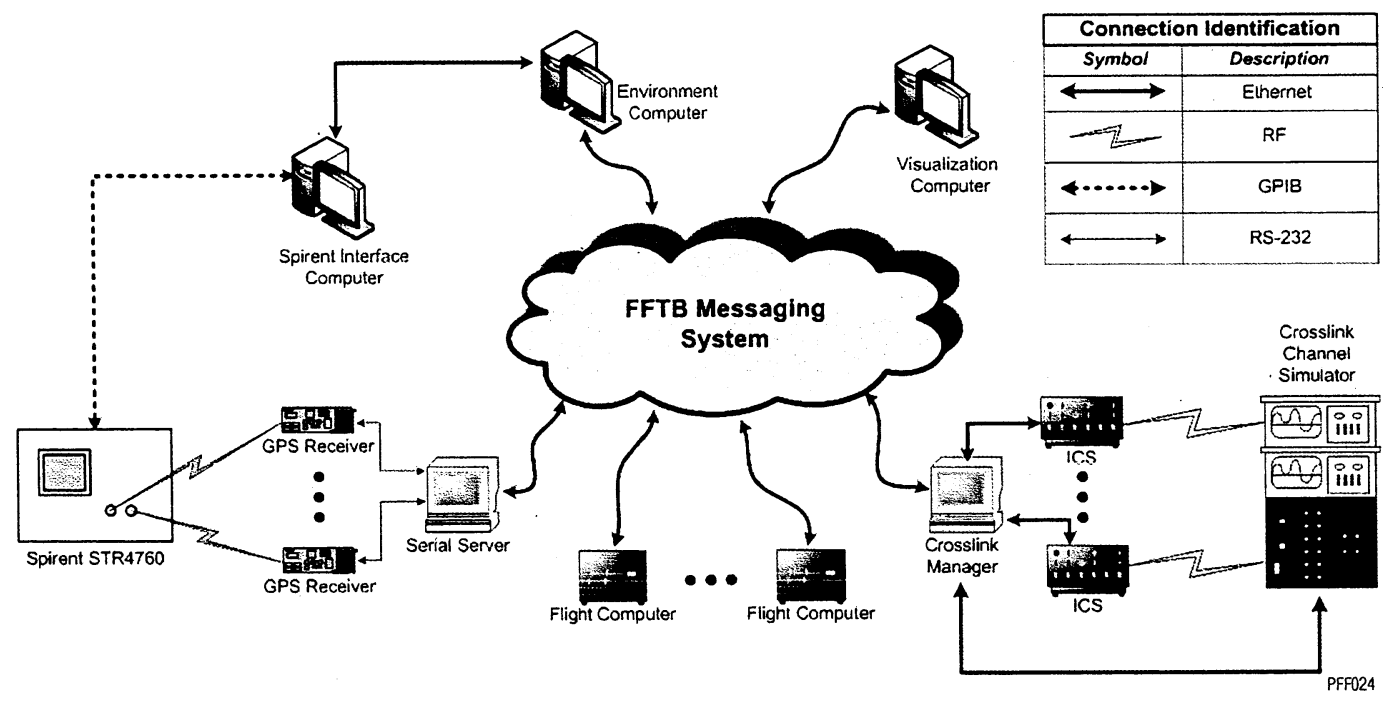

Figure 8. Ground Implementation of ST9 PFF

The spacecraft dynamics and the single spacecraft GNC functions are well-known, and implemented in software simulations. The GPS receivers and ICS devices are incorporated in hardware form, using Spirent GPS signal simulators and a crosslink channel simulator, the GSFC Path Emulator for RF Signals (PERFS), to represent the realistic environmental effects on the radio frequency $(R F)$ signals, such as delay, Doppler, attenuation, and ionospheric effects.

\section{Conclusion}

If selected through an appropriate mission opportunity, the aforementioned Precision Formation Flying (PFF) mission will be the first demonstration of continuous, closed-loop, precise control of multiple spacecraft geometry implemented through 1), co-launched on the SI9-provided LV carrying a Formation Flying System (FFS), including algorithms, sensors, and RF communication to enable a series of maneuvering and proximity operations experiments over a 1-month experiment period. The FFS is currently at a system level TRL of 4, consistent with New

\section{Your launch vehicle is not likely to be ST9-provided at this point.}


- Millennium requirements. The Formation and Attitude Control System (FACS), the software package that encompasses the formation flying algorithms, is completely scalable to future science missions, as is the coarse ranging function that will be added to the intersatellite communication system (ICS) in Phase B. Facilities at the Goddard Space Flight Center (GSFC) and the Jet Propulsion Laboratory (JPL) will be used to demonstrate TRL 6 for the FFS prior to launch. The Formation Flying Testbed (FFTB) at the GSFC will stimulate the GPS and communication- in-the-loop aspects using ICS and GPS hardware- in-theloop, while the JPL Formation Flying Technology facilities will handle implementation on the distributed flight processing architecture. ST9 PFF is the only complete mission concept under consideration for flight prior to the planned NASA strategic PFF-enabled mission concepts, namely the Terrestrial Planet FinderInterferometer (TPF-I), Stellar Imager (SI), Black Hole Imager (BHI), Life Finder (LF), and Planet Imager (PI).

\title{
Appendix-PFF Experiment Primitives
}

\begin{abstract}
foldouf dest escribes the five PFF experiment primitives. For nominal formation operations, there are three basic formation functions: hold; reconfigure; and synchronized rotation, which is a rotation of the formation as a virtual rigid body that synchronizes spacecraft positions and attitudes. There are also two primitives associated with off-nominal functions: formation acquisition and reactive collision avoidance. During each experiment, exactly one spacecraft will be constrained to not perform translational maneuvers. All spacecraft will always control their attitudes. Although two-spacecraft formations are used as examples, the experiments are applicable to larger formations.
\end{abstract} riment primitives are the building blocks from which all the experiments are constructed. This

\section{Formation Acquisition}

In orbital regimes where GPS is not feasible, inertial position knowledge is not accurate enough to determine relative position for spacecraft separated by less than several kilometers. In any regime, near or far from Earth, before relative position control can occur, the spacecraft must establish communication links and locate each other using on-board, relative sensors. In general, the spacecraft must execute rotational and possibly translational maneuvers to acquire communication links and relative sensor lock. Since the spacecraft are drifting with respect to one another, these maneuvers constitute a coupled translational/rotational guidance problem. GU (i.e., interplanetary or in weak gravitational environments) formation acquisition algorithms generally assume spacecraft travel along linear paths unless a translational thruster impulse is delivered. Hence, GU algorithms are not guaranteed to result in a successful acquisition in the near-Earth environment. On the other hand, GC (i.e., regimes with strong central body gravitation or other significant multi-body gravitational effects) formation acquisition requires higher fidelity assumptions with regard to relative motion. For the LEO GC case, GPS with a crosslink is sufficient for formation acquisition as long as the GPS antennas provide adequate coverage.

\section{Formation Hold}

A formation must be able to hold a constant shape and orientation with respect to a given reference frame $\mathrm{F}$. This formation capability is necessary both for stop-and-stare distributed aperture science and for formationkeeping between science observations. Holds may also be only in translational degrees of freedom, referred to as a 3 DOF Hold, or in all degrees of freedom, which is referred to as a 6 DOF Hold. 3 DOF and 6 DOF Holds have also been referred to as Basic Observation and Observation with Co-pointing. GU experiments assume $\mathrm{F}$ is an Inertial frame. Then a 3 DOF Formation Hold is equivalent to maintaining $\mathrm{N}-1$ relative position vectors constant in an inertial frame, where the vectors represent the position of the spacecraft with respect to a reference spacecraft in the formation. GC experiments assume F is a Local Vertical Local Horizontal (LVLH) frame. There is an additional subtlety for GC formations. These formations often maintain passive relative orbits (PROs), which are thrust-free periodic relative motions in an LVLH frame in the absence of navigation errors and external perturbation inclu ding J, differential drag, etc. Although a spacecraft on a PRO does not have a constant relative position vector, a relative trajectory is being held. Hence, for the purposes of classification, we also consider maintaining a PRO to be a formation hold. During a GU Hold, relative motion as viewed in the LVLH coordinate frame resembles the offset circle shown in Figure 9. 

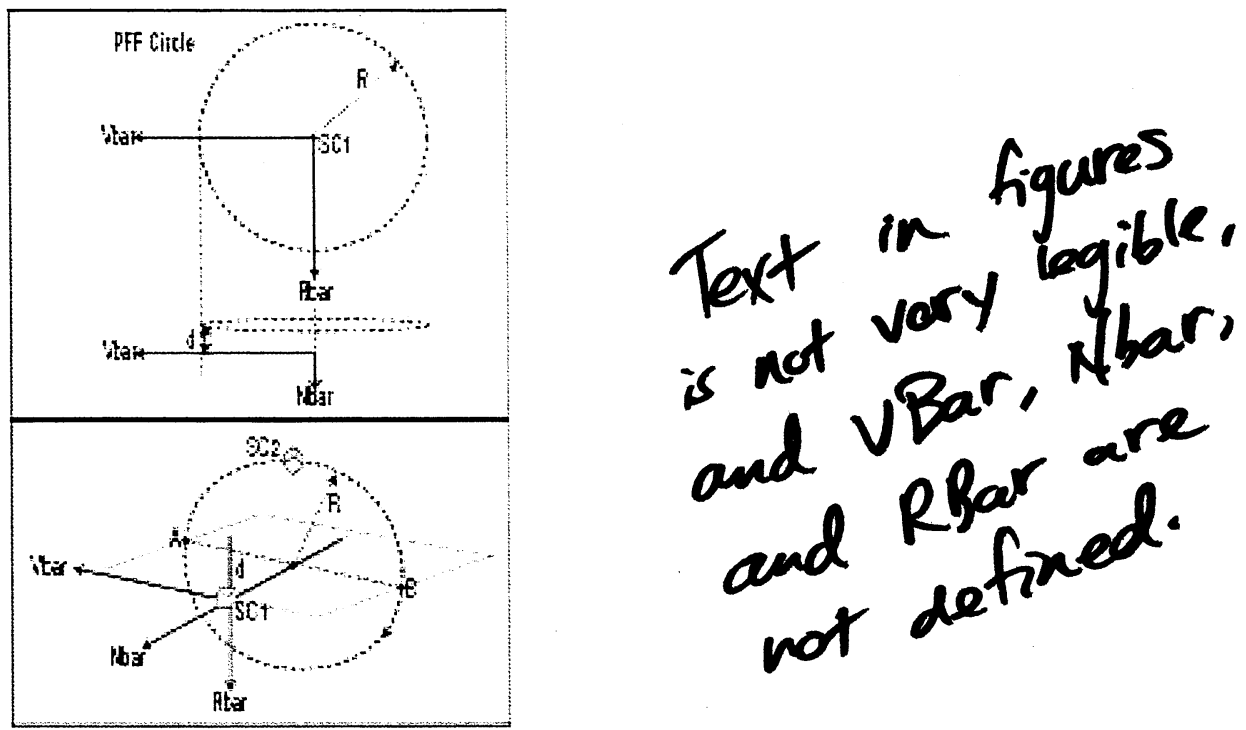

Figure 9. GU Hold

$\mathrm{SC} 1$ is assumed to be the non-maneuvering spacecraft. Every other spacecraft in the formation prescribes an offset circle in the LVLH frame. An offset circle is parameterized by two quantities the radius $R$ and the offset $d$. These parameters are also convenient for describing a safe parking orbit (SPO). Figure 10 shows an example Formation Hold experiment sequence.

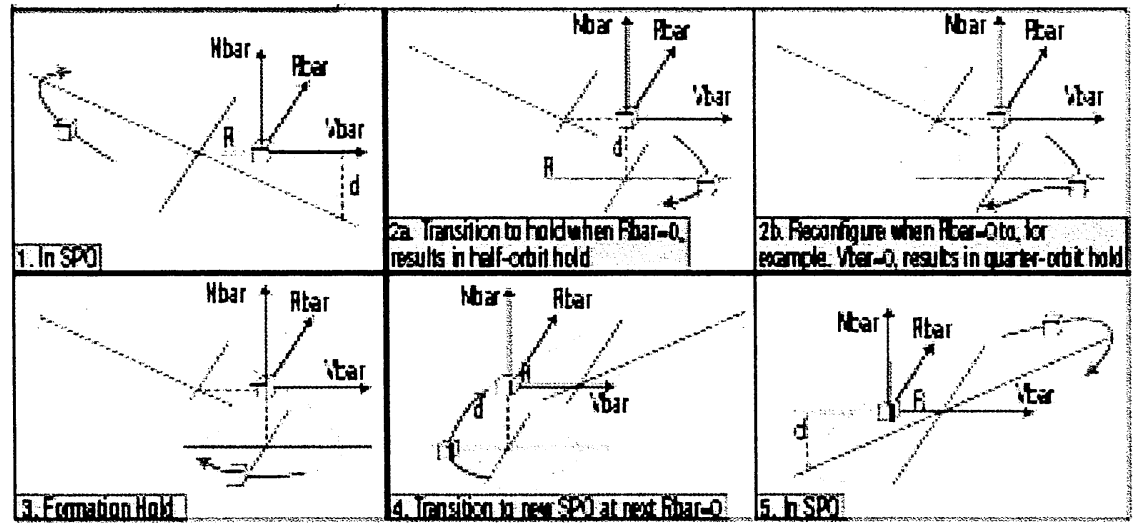

Figure 10. GU Hold Entry/Exit Sequence

A formation hold state will be entered in one of two ways: a) the formation is at or very near a desired formation hold state at the start of the experiment (point A or B in Figure 9 as shown in Figure 10, step 2a), or $\mathrm{b}$ ) the formation reconfigures to a desired formation hold state as shown in step $2 \mathrm{~b}$ and as described in reconfigurations in the following column. During a Formation Hold, the attitude of the formation spacecraft is constrained such that the spacecraft maintain the desired formation sensing topology. For a 2-spacecraft formation, the spacecraft simply point their relative sensors boresights at each other. This constraint fixes two of the three rotational degrees of freedom. The roll about the relative sensors boresight is not constrained and can be used, for example, to improve the solar panel pointing.

\section{Reconfigurations}

A reconfiguration is a transition from one set of relative trajectories to a new set. There are several reasons for demonstrating reconfigurations. First, for general stop-and-stare distributed aperture science in deep space, reconfigurations are necessary to move from one science baseline (i.e., formation hold) to another. Several baseline orientations and magnitudes are generally required to fill the so-called uv-plane for science. 
Reconfigurations are also needed after spacecraft deployment to move the spacecraft to an initial science configuration. Finally, reconfigurations can be used to retarget an observatory formation; for example, to change the observation plane of a GU formation or the PRO of a GC formation. A reconfiguration algorithm plans relative trajectories for all formation spacecraft to transition from their current state to the new, desired relative trajectories. The new trajectories are considered given. The transition trajectories are collision-free and minimize a formation-wide metric, such as total fuel consumed. As a specific example, consider a change in baseline. This change generally requires a translation and rotation of every spacecraft in a formation. For

C ST9 only one spacecraft will exercise translation control during a reconfiguration. During a reconfiguration it is possible to lose formation sensor lock for brief periods of time. This loss is acceptable as long as the $\mathcal{Q}$ error variance of the relative position estimates remain tolerable (i.e., the no-lock pe riods) are short and infrequent). For ST9, reconfigurations can also be used to establish an initial formation hold state (as step $2 \mathrm{~b}$ of Figure 10 shows). Figure 11 shows an example GU reconfiguration as viewed in the LVLH frame where a baseline is doubled while keeping the same baseline orientation in inertial space.

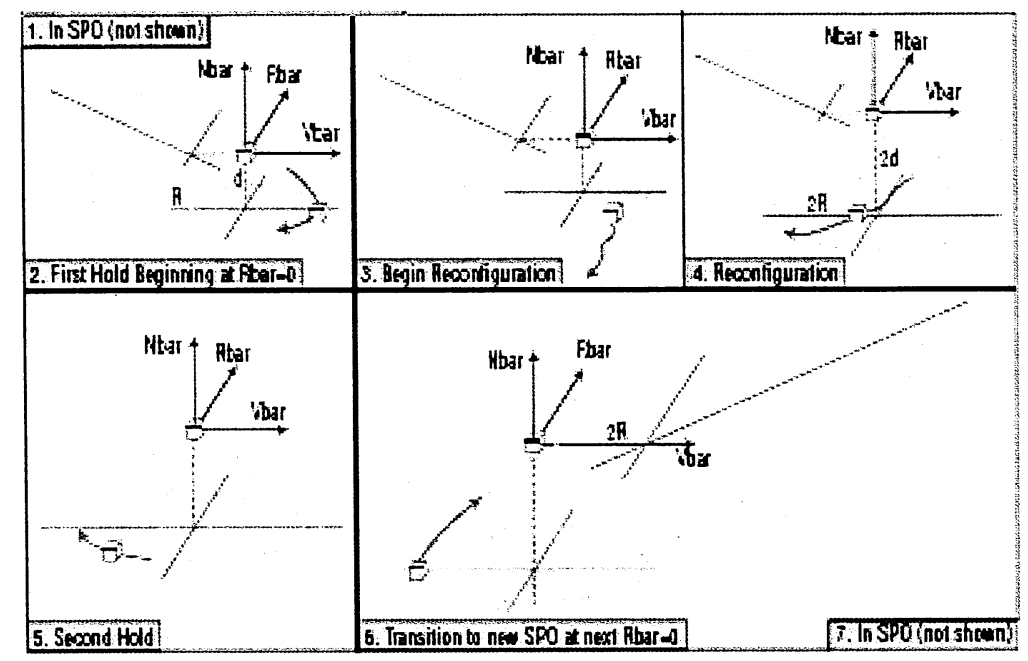

Figure 11. Reconfiguration for change in baseline

A formation hold is established before and after the reconfiguration, emulating a stop-and-stare observation. In the above example, the nominal relative trajectory is trivially free of collision. Reconfigurations will also be demonstrated where the unconstrained, energy-optimal reconfiguration would result in the penetration of a large protection region around the other spacecraft to simulate a collision. The reconfiguration algorithm will recognize this condition and re-plan the reconfiguration to avoid penetration of the protection region. The protection region will be defined conservatively so that an actual collision is very unlikely under expected worst-case disturbances and errors. For example, if the protection region were to be a $50 \mathrm{~m}$ sphere around one of the spacecraft, the unconstrained reconfiguration could be targeted to result in a $40 \mathrm{~m}$ separation. All such maneuvers will be scheduled so as to allow for near-continuous monitoring and over-ride by the ground.

\section{Synchronized Rotation}

During a synchronized rotation, the formation rotates as a single rigid entity about a specified inertial axis. In particular, spacecraft attitudes are synchronized with relative positions. This formation motion is useful for both planet detection and planet characterization (e.g., observation on-the-fly). GC formations can demonstrate a similar maneuver wherein spacecraft travel on a special near-circular PRO and synchronize attitudes with position. GU Synchronized Rotations will be the highest consumer of propellant. Furthermore, in the near-Earth environment, the choice of inertial rotation direction and duration and rate of rotation has significant fuel consumption implications. Synchronized Rotations have also been referred to as Observation with Rotation. 


\section{. Reactive Collision Avoidance}

The purpose of formation guidance is to plan motions that avoid collisions. Still, as a practical necessity, any formation flying mission will be required to have a Reactive Collision Avoidance (RCA) capability to accommodate off-nominal situations. For reactive collision avoidance, all spacecraft in the formation continuously remain in a collision detection state. Once an imminent threat of collision is detected, the RCA system plans and executes appropriate evasive actions. To demonstrate this capability, one or more spacecraft in the formation will be placed purposely on trajectories that penetrate a protection region around one of the spacecraft, as discussed above.

\section{Acknowledgments}

The authors would like to thank the formation flying and systems engineering teams at GSFC and JPL, NASA's New Millennium Program for funding and programmatic support, the Technology Review Board, led by Jack Stocky of JPL, as well as Mark Edison and other supporting staff at Orbital Sciences Corporation for support to the concept development and for some of the graphics work in this paper.

\section{References}

\footnotetext{
${ }^{1} \mathrm{M}$ Oda. Experiences and lessons learned from the ETS-VII robot satellite. In Proceedings of the 2000 IEEE International Conference on Robotics and Automation, pages 914-919, 2000.

${ }^{2}$ David Folta and Albin Hawkins. Preliminary results of NASA's first autonomous formation flying experiment: Earth Observing-1 (EO-1). In Flight Mechanics Symposium, Goddard Space Flight Center, Greenbelt, MD, 2001.

${ }^{3}$ M. Kirschner, O. Montenbruck, and S. Bettadpur. Flight dynamics aspects of the grace formation flying. In 2nd International_Warkaban on Satellite Constellations and_Formation Flyinq. Haifa Isanel February 2001. CNES.

4 Mission Engineering Goddard Space Flight Center and Systems Analysis Division. GEONS open architecture solutions for onboard orbit determination in any orbit. nttp:7/geons.gstc.ntasta.gov.

${ }^{5}$ D. P. Scharf, F. Y. Hadaegh, Z. H. Rahman, J. F. Shields, G. Singh, and M. R. Wette. An overview of the formation and attitude control system for the terrestrial planet finder interferometer. In 2nd International Symposium on Formation Flying Missions and Technologies, 2004.

${ }^{6}$ Russell P. Patera. Satellite collision probability for nonlinear relative motion. Journal of Guidance, Control, and Dynamics, 26(5):728-733, September-October 2003.

${ }^{7}$ B. Lurie. Multi-mode synchronized control for a formation flying interferometer. In AIAA Guidance, Navigation and Control Conference, 2003.
}

\section{Shoull be Goddord Space Fight Center Mission Enginaering and Systew Analysis Dinsion.}


Table 1. Experiment Table

\begin{tabular}{|c|c|c|c|c|c|c|c|c|c|c|c|c|c|c|}
\hline & \multirow[b]{2}{*}{ Basic Description } & \multirow[b]{2}{*}{ Duration } & \multirow[b]{2}{*}{$\begin{array}{l}\text { Relative } \\
\text { Geometry }\end{array}$} & \multicolumn{4}{|c|}{ Autonomy } & \multirow[b]{2}{*}{$\begin{array}{l}\text { 음 } \\
\text { 옹 } \\
\text { 음 } \\
\text { 흠 }\end{array}$} & \multicolumn{4}{|c|}{ PFF Primitive } & \multirow[b]{2}{*}{$\begin{array}{l}\text { 몷 } \\
\text { 윯 } \\
\text { 들 } \\
\text { 퐁 } \\
\text { 요 }\end{array}$} & \multirow[b]{2}{*}{ 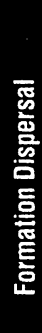 } \\
\hline & & & & 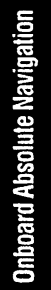 & 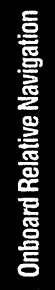 & 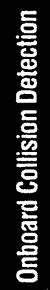 & 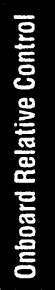 & & 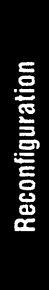 & 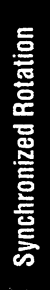 & 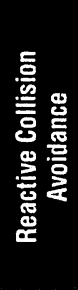 & 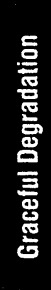 & & \\
\hline $\begin{array}{l}\text { Separation } \\
\text { and } \\
\text { Checkout }\end{array}$ & $\begin{array}{l}\text { Stack separation, } \\
\text { checkout individual } \\
\text { and formation level } \\
\text { spacecraft systems }\end{array}$ & 2 weeks & $\begin{array}{l}\text { In-track and } \\
\text { cross sep }\end{array}$ & $x$ & & & & & & & & & & \\
\hline Parking & $\begin{array}{l}\text { Pause for data dump, } \\
\text { prepare for next } \\
\text { experiment }\end{array}$ & 3-6 days & $\begin{array}{l}1 \mathrm{~km} \text { offset, } \\
300 \times 600 \times 300 \mathrm{~m} \\
\text { safety ellipse } \\
\text { (SE) }\end{array}$ & $x$ & $x$ & $x$ & & & & & & & & \\
\hline Staging & $\begin{array}{l}\text { Maneuver to pre-exp. } \\
\text { Geometry }\end{array}$ & $1-5$ revs & Walking SE & $x$ & $x$ & $x$ & $x$ & & & & & & $x$ & \\
\hline Dispersal & $\begin{array}{l}\text { Maneuver to } \\
\text { permanently separated } \\
\text { orbits }\end{array}$ & . & & $x$ & $x$ & $x$ & & & & & & & & $x$ \\
\hline GC EXP & $\begin{array}{l}\text { Precisely maintain } \\
\text { near-natural motion }\end{array}$ & 3 revs & $\begin{array}{l}\text { Near natural SE } \\
\text { motion w/out } J 2 \\
\text { accommodation }\end{array}$ & $x$ & $x$ & $x$ & $x$ & $x$ & & & & & & \\
\hline GC EXP & $\begin{array}{l}\text { Precisely maintain } \\
\text { near-natural motion }\end{array}$ & 3 revs & $\begin{array}{l}\text { Near natural SE } \\
\text { motion w/ J2 } \\
\text { accommodation }\end{array}$ & $x$ & $x$ & $x$ & $x$ & $x$ & & & & & & \\
\hline GC EXP & $\begin{array}{l}\text { Simulated system } \\
\text { failure }\end{array}$ & 3 revs & $\begin{array}{l}\text { Near natural } \\
\text { SE motion, } \\
\text { formation safing } \\
\text { maneuver }\end{array}$ & $x$ & $x$ & $x$ & $x$ & $x$ & & & & $x$ & $x$ & \\
\hline GUEXP & $\begin{array}{l}\text { Precisely maintain } \\
\text { inertial hold }\end{array}$ & 0.5 revs & $\begin{array}{l}\text { inertial hold }(\mathrm{IH}) \\
\text { at max out-of- } \\
\text { plane offset }\end{array}$ & $x$ & $x$ & $x$ & $x$ & $x$ & & & & & & \\
\hline GUEXP & $\begin{array}{l}\text { Perform reconfiguration } \\
\text { between precision } \\
\text { inertial holds }\end{array}$ & 0.5 revs & $\begin{array}{l}\mathrm{IH} \text {, then expand } \\
\text { baseline to a } \\
\text { second } \mathrm{IH}\end{array}$ & $x$ & $x$ & $x$ & $x$ & $x$ & $x$ & & & & & \\
\hline GUEXP & $\begin{array}{l}\text { Simulate synchronized } \\
\text { rotation about an } \\
\text { inertial axis }\end{array}$ & 0.5 revs & $\begin{array}{l}\text { Rotate as rigid } \\
\text { formation about } \\
\text { inertial axis }\end{array}$ & $x$ & $x$ & $x$ & $x$ & $x$ & $x$ & $x$ & & & & \\
\hline GUEXP & $\begin{array}{l}\text { Safely test reactive } \\
\text { collision avoidance }\end{array}$ & 0.5 revs & $\begin{array}{l}\text { Safe trajectories } \\
\text { which trigger } \\
\text { conservative } \\
\text { collision alarm }\end{array}$ & $x$ & $x$ & $x$ & $x$ & $x$ & $x$ & & $x$ & & $x$ & \\
\hline GUEXP & $\begin{array}{l}\text { Perform sequence } \\
\text { of autonomous } \\
\text { observations }\end{array}$ & 0.5 revs & $\begin{array}{l}\text { Sync. rotation, } \\
\text { reconfigure, } \\
\text { sync. rotation }\end{array}$ & $x$ & $x$ & $x$ & $x$ & $x$ & $x$ & $x$ & & & & \\
\hline
\end{tabular}

